\title{
Monocyte-to-serum albumin Ratio as a Novel Predictor of Long-Term Adverse Outcomes in Patients after Undergoing Percutaneous Coronary Intervention: A Retrospective Cohort Study
}

Ying-Ying Zheng ( $\square$ zhengying527@163.com )

Zhengzhou University First Affiliated Hospital https://orcid.org/0000-0003-0003-4270

\section{Zeng-Lei Zhang}

Zhengzhou University First Affiliated Hospital

Qian-Qian Guo

Zhengzhou University First Affiliated Hospital

Jun-Nan Tang

Zhengzhou University First Affiliated Hospital

\section{Xu-Ming Yang}

Henan University of Science and Technology Affiliated First Hospital

Jian-Chao Zhang

Zhengzhou University First Affiliated Hospital

Meng-Die Cheng

Zhengzhou University First Affiliated Hospital

\section{Feng-Hua Song}

Zhengzhou University First Affiliated Hospital

\section{Zhi-Yu Liu}

Zhengzhou University First Affiliated Hospital

\section{Kai Wang}

Zhengzhou University First Affiliated Hospital

\section{Li-Zhu Jiang}

Zhengzhou University First Affiliated Hospital

\section{Lei Fan}

Zhengzhou University First Affiliated Hospital

Xiao-Ting Yue

Zhengzhou University First Affiliated Hospital

\section{Yan Bai}

Zhengzhou University First Affiliated Hospital

Xin-Ya Dai

Zhengzhou University First Affiliated Hospital 


\section{Ru-Jie Zheng}

Zhengzhou University First Affiliated Hospital

\section{Jin-Ying Zhang}

Zhengzhou University First Affiliated Hospital

\section{Research}

Keywords: monocyte-to-serum-albumin ratio, percutaneous coronary intervention, mortality, prognosis.

Posted Date: November 18th, 2020

DOl: https://doi.org/10.21203/rs.3.rs-109729/v1

License: (c) (i) This work is licensed under a Creative Commons Attribution 4.0 International License. Read Full License 


\section{Abstract}

Background: Inflammation plays a significant role in the initiation and progression of atherosclerosis. Monocyte and serum albumin have been proved to be involved in the process of systemic inflammation. Therefore, we investigated the prognostic value of monocyte-to-serum albumin ratio (MAR) in patients who underwent percutaneous coronary intervention (PCl).

Methods $\mathbb{\text { A total }}$ of 3,561 patients enrolled in this study from January 2013 to December 2017, who were divided into two groups according to the cut-off value of MAR (MAR<0.014, n=2220 and MAR $\geq 0.014$, $n=1119)$. The average follow-up time was $37.59 \pm 22.24$ months.

Results: There were significant differences between the two groups in the incidences of all-cause mortality (ACM) $(P<0.001)$, cardiac mortality $(\mathrm{CM})(P<0.001)$, major adverse cardiovascular events (MACEs, $P=0.038$ ) and major adverse cardiovascular and cerebrovascular events (MACCEs, $P=0.037$ ). Kaplan-Meier survival analysis suggested that patients with higher MAR value tended to have an increased accumulated risk of ACM and CM (Log rank $P<0.001$ and Log rank $P<0.001$, respectively). And multivariate Cox regression analyses showed MAR was an independent predictor for ACM (hazard ratio $[H R]=1.461,95 \%$ confidence interval $[C l: 1.009-2.115, P=0.045)$ and $C M(H R=1.695,95 \% C l: 1.056-2.721$, $P=0.029)$.

Conclusion: The present study suggests that MAR is a novel independent predictor of long-term mortality in patients who underwent $\mathrm{PCl}$.

Trial registration: ChiCTR, ChiCTR1800019699. Registered 24 November 2018, http://www.chictr.org.cn/showproj.aspx?proj=33249

\section{Background}

Over the past few decades, percutaneous coronary intervention $(\mathrm{PCl})$ has revolutionized the management of coronary heart disease (CHD), with a significant mortality reduction [1]. However, the long-term mortality benefit of $\mathrm{PCl}$ has not been shown in these patients and the prognostic benefits still remain uncertain [2]. Consequently, a long-term risk stratification in patients treated with primary PCI is very important to help guide management decisions in these patients.

The role of inflammation in the pathogenesis and prognosis of $\mathrm{CHD}$ has gained wide attention [3]. Recently, inflammation based indexes, such as monocyte to high-density lipoprotein cholesterol ratio, platelet-to-lymphocyte ratio (PLR) and C-reactive protein-to-albumin ratio, have been reported as useful prognostic indicators in patients with $\mathrm{CHD}[4-6]$.

A large number of evidences implicate that monocyte plays an important role in the progression of atherosclerosis, by interacting with platelets and leading to chronic activation of pro-inflammatory leukocyte subpopulations $[7,8]$. Berg et.al showed that the percentage and number of classical 
monocytes could predict cardiovascular events [9]. As a negative acute phase protein, serum albumin is considered as the intensity of the infection-triggered inflammatory response [10]. Population-base studies have shown that the lower serum albumin is associated with the development of atherosclerosis, and the initiation of myocardial infarction $[11,12]$.

Considerably, the monocyte and serum albumin are associated with the progression of CHD. However, the value of monocyte to albumin ratio (MAR) in CHD patients after $\mathrm{PCl}$ has not been investigated. Therefore, we investigated the prognostic value of MAR in patients who underwent PCl.

\section{Methods}

\subsection{Participants}

A total of 3,561 patients with CHD who underwent PCl at the First Affiliated Hospital of Zhengzhou University between January 2013 to December 2017 were enrolled. The inclusion criteria were CHD patients who were confirmed by coronary angiography, including non-ST-segment elevation acute coronary syndrome (ACS), ST-segment elevation ACS and stable angina and implanted at least one stent. Exclusion criteria included severe valvular heart disease, decompensated heart failure, pulmonary heart disease, rheumatic heart disease, serious renal or hepatic disease, pernicious anemia, infective diseases and malignancy. This study complies with the Declaration of Helsinki and was approved by the ethics committee of the First Affiliated Hospital of Zhengzhou University.

\subsection{Follow Up}

Two hundred and twenty-two patients were excluded for the monocyte or albumin data not being available or the presence of renal failure, infective disease and malignancies. Finally, 3,339 patients were evaluated and categorized into two groups by MAR value (MAR $<0.014, n=2220$ and $M A R \geq 0.014, n=$ 1119). The cut-off value of MAR (0.014) was according to the analysis of the ROC curve for the baseline monocyte-to-serum albumin ratio of the study population. The flowchart of the participants' enrollment is shown in Fig. 1. In our center, all patients undergoing PCI received a regular follow up conducted by telephone interview. During the duration of follow up, all events were carefully checked and verified by an independent group of clinical physicians.

\subsection{Outcome}

The primary endpoints included all-cause mortality (ACM) and cardiac mortality (CM). The secondary endpoints were stroke, bleeding, rehospitalization, major adverse cardiovascular events (MACEs) and major adverse cardiac and cerebrovascular events (MACCEs). MACEs were defined as the composite of cardiac death, recurrent myocardial infarction (MI), or target-vessel revascularization. And MACCEs were defined as MACES plus stroke $[13,14]$.

\subsection{Statistical Analysis}


All data was analyzed by the SPSS 22.0 for Windows statistical software (SPSS Inc, Chicago, Illinois, United States). The enrolled patients were grouped into 2 categories based on the value of MAR (MAR< 0.014 and MAR $\geq 0.014$ ). Continuous data were given as the mean \pm standard deviation, and categorical variables were presented as numbers and percentages. To compare the difference of parametric continuous variables, Student's $t$ tests were used; and Mann-Whitney U tests were used when compared the difference of nonparametric continuous variables. Chi-squared $\left(\chi^{2}\right)$ tests were used for the comparison of categorical variables. Kaplan-Meier analysis was used for cumulative incidence rates of long-term outcomes and the log-rank test was employed to compare between groups. Multivariate Cox regression analysis was performed to examine the predictive value of the MAR for outcomes during and up to the 6-year follow-up. Hazard ratios (HRs) and 95\% confidence intervals (Cls) were calculated. The $P$ values were 2 -sided, considered being significant difference when $P<0.05$.

\section{Result}

\subsection{Baseline characteristics}

In the study, 3,339 patients who underwent PCl were enrolled. The median follow-up time is $37.59 \pm$ 22.24months. The participants were categorized into two groups according to the cut-off value of MAR: the lower MAR group (MAR < 0.014, $n=2220$ ) and the higher MAR group (MAR $\geq 0.014, n=1119)$. All the baseline data were shown in Table 1. The variables in regards to age, gender, smoking, heart rate, creatinine (Cr), uric acid (UA) and high-density lipoprotein (HDL-C) showed significant differences between the two groups (all $P<0.05$ ). 
Table 1

Baseline characteristics

\begin{tabular}{|c|c|c|c|c|}
\hline Variables & $\begin{array}{l}\text { MAR }<0.014 \\
(n=2220)\end{array}$ & $\begin{array}{l}\text { MAR } \geq 0.014 \\
(n=1119)\end{array}$ & $x 2$ or $t$ & $P$-value \\
\hline Age, years & $62.74 \pm 10.47$ & $64.37 \pm 10.92$ & -4.189 & $<0.001$ \\
\hline Gender (male), n (\%) & $1441(64.9)$ & $858(76.7)$ & 48.024 & $<0.001$ \\
\hline Family history of CAD, $\mathrm{n}(\%)$ & $425(19.3)$ & $202(18.2)$ & 0.575 & 0.448 \\
\hline Smoking, n (\%) & $620(27.9)$ & $397(35.5)$ & 20.023 & $<0.001$ \\
\hline Alcohol drinking, n (\%) & $340(15.3)$ & $200(17.9)$ & 3.590 & 0.058 \\
\hline Diabetes, n (\%) & $514(23.2)$ & $273(24.4)$ & 0.639 & 0.424 \\
\hline Hypertension, n (\%) & $1242(55.9)$ & $614(54.9)$ & 0.349 & 0.555 \\
\hline Heart rate, rpm & $74.10 \pm 20.38$ & $75.55 \pm 11.92$ & -2.150 & 0.032 \\
\hline Cr, umol/L & $70.39 \pm 24.69$ & $77.69 \pm 51.08$ & -5.547 & $<0.001$ \\
\hline UA, mmol/L & $294.81 \pm 82.33$ & $307.50 \pm 91.96$ & -4.017 & $<0.001$ \\
\hline TG, mmol/L & $1.69 \pm 1.17$ & $1.62 \pm 1.02$ & 1.778 & 0.075 \\
\hline TC, $\mathrm{mmol} / \mathrm{L}$ & $3.92 \pm 1.03$ & $3.86 \pm 1.01$ & 1.608 & 0.108 \\
\hline LDL-C, mmol/L & $2.40 \pm 0.84$ & $2.40 \pm 0.85$ & 0.184 & 0.854 \\
\hline $\mathrm{HDL}-\mathrm{C}, \mathrm{mmol} / \mathrm{L}$ & $1.06 \pm 0.30$ & $1.00 \pm 0.27$ & 4.621 & $<0.001$ \\
\hline
\end{tabular}

\subsection{Comparison Of Clinical Outcomes}

We showed the clinical outcomes in Table 2. For the primary endpoints, the incidence of ACM was $2.9 \%$ in the lower MAR group and 5.5\% in the higher MAR group $(P<0.001)$. And the incidence of CM was a significant difference between the two groups $(1.7 \%$ vs. $3.8 \%, P<0.001)$. For the secondary endpoints, the incidence of MACEs and MACCEs between the two groups showed significant differences $(10.5 \% \mathrm{vs}$. $13.0 \%, P=0.038$ and $13.8 \%$ vs. $16.5 \%, P=0.037$, respectively). However, there were no significant differences between the two groups in regards to the incidence of stroke, bleeding events and readmission. As Kaplan-Meier survival analysis suggested that $\mathrm{CHD}$ patients with higher MAR tended to have an increased accumulated risk of ACM and CM (Log rank $P<0.001$ and Log rank $P<0.001$, respectively) (Fig. 2 and Fig. 3). 
Table 2

Outcomes comparison between two groups

\begin{tabular}{|lllll|}
\hline Variables & $\begin{array}{l}\text { MAR }<0.014 \\
(\mathbf{n}=2220)\end{array}$ & $\begin{array}{l}\text { MAR } \geq 0.014 \\
(\mathbf{n = 1 1 1 9})\end{array}$ & $\chi 2$ or $\boldsymbol{t}$ & P-value \\
\hline Primary endpoints & & & & \\
\hline ACM, $\mathrm{n}(\%)$ & $65(2.9)$ & $62(5.5)$ & 13.881 & $<0.001$ \\
\hline CM, $\mathrm{n}(\%)$ & $37(1.7)$ & $42(3.8)$ & 14.024 & $<0.001$ \\
\hline secondary endpoints & & & & \\
\hline MACEs, $n(\%)$ & $234(10.5)$ & $145(13.0)$ & 4.321 & $\mathbf{0 . 0 3 8}$ \\
\hline MACCEs, $n(\%)$ & $307(13.8)$ & $185(16.5)$ & 4.329 & $\mathbf{0 . 0 3 7}$ \\
\hline
\end{tabular}

\subsection{Prognostic significance of MAR in patients who underwent $\mathrm{PCI}$}

Baseline variables, which showed significant differences $(P<0.05)$ between two groups in univariate models entered into multivariate Cox regression analysis. In the multivariate Cox regression analysis, compared with the lower MAR group, the risk of ACM, CM in the higher group were increased by $46.1 \%$ $(\mathrm{HR}=1.461,95 \% \mathrm{Cl} .1 .009-2.115, P=0.045), 69.5 \%$ (HR=1.695, 95\% Cl.1.056-2.721, $P=0.029)$ respectively (Tables 3 and 4 ). We did not find significant difference in the MACEs (HR $=1.147,95 \%$ Cl:0.921-1.428, $P=0.220)$ and MACCEs (HR $=1.154,95 \%$ Cl:0.952-1.399, $P=0.144)$ between the two groups.

Table 3

Cox regression analysis results for ACM

\begin{tabular}{|llllll|}
\hline Variables & $\boldsymbol{B}$ & SE & Wald & $\boldsymbol{P}$ & $H R(95 \% \mathrm{CI})$ \\
\hline Age [years] & 0.069 & 0.010 & 51.355 & $<0.001$ & $1.072(1.052-1.092)$ \\
\hline Gender (male) & -0.176 & 0.239 & 0.544 & 0.461 & $0.838(0.525-1.340)$ \\
\hline Smoking & 0.056 & 0.218 & 0.066 & 0.797 & $1.058(0.690-1.622)$ \\
\hline Heart rate & 0.002 & 0.002 & 1.130 & 0.288 & $1.002(0.998-1.007)$ \\
\hline Cr & 0.007 & 0.001 & 53.038 & $<0.001$ & $1.007(1.005-1.008)$ \\
\hline HDL-C & -0.530 & 0.370 & 2.048 & 0.152 & $0.589(0.285-1.216)$ \\
\hline UA & 0.001 & 0.001 & 0.317 & 0.573 & $1.001(0.999-1.003)$ \\
\hline MAR & 0.379 & 0.189 & 4.023 & 0.045 & $1.461(1.009-2.115)$ \\
\hline
\end{tabular}


Table 4

Cox regression analysis results for $\mathrm{CM}$

\begin{tabular}{|llllll|}
\hline Variables & $\boldsymbol{B}$ & SE & Wald & $\boldsymbol{P}$ & HR $(95 \% \mathrm{Cl})$ \\
\hline Age [years] & 0.064 & 0.012 & 28.382 & $<0.001$ & $1.066(1.041-1.091)$ \\
\hline Gender (male) & -0.531 & 0.340 & 2.444 & 0.118 & $0.588(0.302-1.144)$ \\
\hline Smoking & 0.110 & 0.263 & 0.174 & 0.676 & $1.116(0.667-1.868)$ \\
\hline Heart rate & 0.002 & 0.003 & 0.282 & 0.596 & $1.002(0.996-1.007)$ \\
\hline Cr & 0.006 & 0.001 & 22.125 & $<0.001$ & $1.006(1.003-1.008)$ \\
\hline HDL-C & -0.874 & 0.489 & 3.199 & 0.074 & $0.417(0.160-1.087)$ \\
\hline UA & 0.002 & 0.001 & 2.253 & 0.133 & $1.002(0.999-1.004)$ \\
\hline MAR & 0.528 & 0.241 & 4.780 & 0.029 & $1.695(1.056-2.721)$ \\
\hline
\end{tabular}

\section{Discussion}

In the present study, we found the prognostic value of MAR in patients who underwent $\mathrm{PCl}$ and demonstrated higher MAR value was independently associated with adverse outcomes of patients who underwent $\mathrm{PCI}$.

Considering the high prevalence and poor prognosis of $\mathrm{CHD}$, the early diagnostic biomarkers for patients with $\mathrm{CHD}$ are in need. Vascular inflammation plays a crucial role in the progression of CHD, and inflammation-based biomarkers have been shown previously [15-17], such as monocyte [18], highsensitivity C-reactive protein and serum albumin [19]. However, it is still elusive which cell plays a role in initiation of the cascade processes.

Atherosclerosis has long been associated with chronic inflammation, and monocytes and monocytederived macrophages account for the coronary plaque progression [20]. Circulating monocytes populate plaques and adhere to the dysfunctional endothelial surface via binding to leukocyte adhesion molecules only expressed by abnormal endothelial cells [21,22]. Cao et al have reported that all stages of atherogenesis, from the earliest lesions (fatty strips) to the formation of complex plaque, are associated with macrophages which are considered as a driving force [23]. In another recent study, efficient degradation of CCR2 mRNA in monocytes attenuated the number of monocytes in atherosclerotic plaques, reduced the infarct size following coronary artery occlusion [24], which may be a novel therapeutic strategy for CHD patients undergoing PCl. Serum albumin is considered as a negative acutephase protein in the progression of inflammation [25]. As previous studies demonstrated, low serum albumin concentration can predict the clinical outcome of acute myocardial infarction [12]. As shown in Table 1, age, gender, smoking, $\mathrm{Cr}$ and UA have significant differences between the 2 groups. Aging, gender 
and smoking are often characterized by a number of dysfunctional conditions including: myocardial sarcopenia, hypertrophy, vascular hyperpermeability, hypertension, inflammation, and functional impairment [26-30]. Increasing evidence demonstrates that age is a major risk factor in cardiac-related morbidity and mortality resulting from the decrease of the self-renewal of yolk sac-derived resident macrophages that play a key role in the post-MI repair and maintaining the cardiac homeostasis with age [31]. Ovaries removed or natural menopausal women exhibit systematic inflammation [32]. CHD has been considered to be male disease [28] because men are at a greater risk of CVD than women [29], who may decrease significantly the secretion of pro-inflammatory markers interleukin-6 (IL-6), IL-1, TNF-a via the estrogen [33]. Smoking has been known as an independent risk factor of atherosclerosis via promoting inflammation, thrombosis, and oxidative stress [30]. There are no doubts that age, gender, smoking, etc are confounding factors in our study, while after including these confounding factors in the multivariate Cox regression analysis, we still obtained positive results which demonstrated that the high MAR is an independent risk factor for $\mathrm{ACM}$ and $\mathrm{CM}$ in patients who underwent $\mathrm{PCl}$.

To the best of our knowledge, it is the first time to demonstrate the MAR, which is a novel, powerful, inexpensive and effective predictor for outcomes of $\mathrm{CHD}$ after $\mathrm{PCl}$. There are some limitations in our study. Firstly, we did not monitor the dynamic change of their variables. Secondly, our study is a single retrospective cohort design. Thirdly, we failed to collect inflammatory markers such as high sensitive $C$ reaction protein (hsCRP) and erythrocyte sedimentation rate (ESR). Besides, to demonstrate the association between MAR and the outcomes of CHD after PCl, our study needs to be further verified by a multi-center, prospective study. And we expect more researches about the mechanism of MAR will be explored.

\section{Conclusion}

In conclusion, our findings suggest that the MAR is a sensitive, reliable and effective predictor of adverse outcomes in $\mathrm{CHD}$ patients undergoing $\mathrm{PCl}$.

\section{Abbreviations}

PCl: percutaneous coronary intervention

MAR: monocyte-to-serum albumin ratio

ACM: all-cause mortality

CHD』coronary heart disease

MACEs $\square$ major adverse cardiovascular events

MACCEs $\square m a j o r$ adverse cardiac and cerebrovascular events 


\section{Declarations}

Acknowledgements

Not applicable.

\section{Authors' contributions}

All of the authors were actively involved in the study. JY Zhang and YY Zheng conceived the study and participated in the design. ZL Zhang, YY Zheng and JN Tang participated in the design, XT Yue, QQ Guo, JC Zhang, MD Cheng, FH Song, ZY Liu, K Wang, LZ J, XM Yang, L Fan, Y Bai, XY Dai and RJ Zheng collected the data, ZL Zhang and YY Zheng performed statistical analyses and drafted the manuscript. JY Zhang and YY Zheng critically reviewed the manuscript. All authors read and approved the final manuscript.

\section{Funding}

This research was funded by the National Natural Science Foundation of China $(81870328,81760043$ and 81800267).

\section{Availability of data and materials}

The datasets used and/or analyzed during the current study are available from the corresponding author on reasonable request.

\section{Ethics approval and consent to participate}

This study complies with the Declaration of Helsinki and was approved by the ethics committee of the First Affiliated Hospital of Zhengzhou University.

\section{Consent for publication}

Not applicable.

\section{Competing interests}

The authors declare that they have no competing interests.

\section{References}

1. Benjamin EJ, Muntner P, Alonso A, Bittencourt MS, Callaway CW, Carson AP, et al. Heart disease and stroke Statistics-2019 update: a report from the American Heart Association. Circulation. 2019;139(10):e56-e528.

2. Weintraub WS, Boden WE, Reexamining the efficacy and value of percutaneous coronary intervention for patients with stable ischemic heart disease. JAMA Intern Med. 2016;176(8):1190-4. 
3. McPherson R, Davies RW, Inflammation and coronary artery disease: insights from genetic studies. Can J Cardiol. 2012;28(6):662-6.

4. Wu TT, Zheng YY, Chen Y, Yu ZX, Ma YT, Xie X. Monocyte to high-density lipoprotein cholesterol ratio as long-term prognostic marker in patients with coronary artery disease undergoing percutaneous coronary intervention. Lipids Health Dis. 2019;18(1):180.

5. Bressi E, Mangiacapra F, Ricottini E, Cavallari I, Colaiori I, Di Gioia G, et al. Impact of neutrophil-tolymphocyte ratio and platelet-to-lymphocyte ratio on 5-year clinical outcomes of patients with stable coronary artery disease undergoing elective percutaneous coronary intervention. J Cardiovasc Transl Res. 2018;11(6):517-523.

6. Wang W, Ren D, Wang CS, Li T, Yao HC, Ma SJ. Prognostic efficacy of high-sensitivity C-reactive protein to albumin ratio in patients with acute coronary syndrome. Biomark Med. 2019;13(10):811820.

7. Frangogiannis NG. Monocyte subsets as predictors of adverse events in patients with atherosclerosis. Hellenic J Cardiol. 2019;60(5):322-323.

8. Kapellos TS, Bonaguro L, Gemünd I, Reusch N, Saglam A, Hinkley ER, et al. Human monocyte subsets and phenotypes in major chronic inflammatory diseases. Front Immunol. 2019;10:2035.

9. Berg KE, Ljungcrantz I, Andersson L, Bryngelsson C, Hedblad B, Fredrikson GN, et al. Elevated CD14++CD16- monocytes predict cardiovascular events. Circ Cardiovasc Genet. 2012;5(1):122-31.

10. Karabağ $Y$, Çağdaş M, Rencuzogullari I, Karakoyun S, Artaç İ, iliş D, et al. Usefulness of the C-reactive protein/albumin ratio for predicting no-reflow in ST-elevation myocardial infarction treated with primary percutaneous coronary intervention. Eur J Clin Invest. 2018;48(6):e12928.

11. Fan Q, Tao R, Zhang H, Xie H, Lu L, Wang T, et al. Dectin-1 contributes to myocardial ischemia/reperfusion injury by regulating macrophage polarization and neutrophil infiltration. Circulation. 2019;139(5):663-678.

12. Plakht $\mathrm{Y}$, Gilutz $\mathrm{H}$, Shiyovich $A$. Decreased admission serum albumin level is an independent predictor of long-term mortality in hospital survivors of acute myocardial infarction. Soroka acute myocardial infarction II (SAMI-II) project. Int. J. Cardiol. 2016;219:20-4.

13. Babapulle MN1, Joseph L, Bélisle P, Brophy JM, Eisenberg MJ. A hierarchical bayesian meta-analysis of randomised clinical trials of drug-eluting stents. Lancet. 2004;364(9434):583-91.

14. Javaid A, Steinberg DH, Buch AN, Corso PJ, Boyce SW, Pinto Slottow TL, et al. Outcomes of coronary artery bypass grafting versus percutaneous coronary intervention with drug-eluting sstents for patients with multivessel coronary artery disease. Circulation. 2007;116(11 suppl):I-200-I-206.

15. Fernández-Friera L, Fuster V, López-Melgar B, Oliva B, Sánchez-González J, Macías A, et al. Vascular inflammation in subclinical atherosclerosis detected by hybrid PET/MRI. J Am Coll Cardiol. 2019;73(12):1371-1382.

16. Teague HL, Ahlman MA, Alavi A, Wagner DD, Lichtman AH, Nahrendorf M, et al. Unraveling vascular inflammation: from immunology to imaging. J Am Coll Cardiol. 2017;70(11):1403-12.

17. Libby P. Inflammation in Atherosclerosis. Nature. 2002;420(6917):868-74. 
18. Ghattas A1, Griffiths HR, Devitt A, Lip GY, Shantsila E. Monocytes in coronary artery disease and atherosclerosis: where are we now? J Am Coll Cardiol. 2013;62(17):1541-51.

19. Don BR, Kaysen G. Serum albumin: relationship to inflammation and nutrition. Seminars in Dialysis. 2004;17(6):432-437.

20. Raggi P, Genest J, Giles JT, Rayner KJ, Dwivedi G, Beanlands RS, et al. Role of inflammation in the pathogenesis of atherosclerosis and therapeutic interventions. Atherosclerosis. 2018;276:98-108.

21. Woollard KJ, Geissmann F. Monocytes in atherosclerosis: subsets and functions. Nature Reviews Cardiology. 2010;7(2):77-86.

22. Libby P, Okamoto Y, Rocha VZ, Folco E. Inflammation in atherosclerosis: transition from theory to practice. Circulation Journal. 2010;74(2):213-220.

23. Cao DJ. Macrophages in Cardiovascular Homeostasis and Disease. Circulation. 2018;138(22):24522455.

24. Leuschner F, Dutta P, Gorbatov R, Novobrantseva TI, Donahoe JS, Courties G, et al. Therapeutic siRNA silencing in inflammatory monocytes in mice. Nat Biotechnol. 2011;29(11):1005-1010.

25. Charlie-Silva I, Klein A, Gomes JMM, Prado EJR, Moraes AC, Eto SF, et al. Acute-phase proteins during inflammatory reaction by bacterial infection: Fish-model. Sci Rep. 2019;9(1):4776.

26. Nevalainen T, Kananen L, Marttila S, Jylhä M, Hervonen A, Hurme M, et al. Transcriptomic and epigenetic analyses reveal a gender difference in aging-associated inflammation: the Vitality $90+$ study. Age (Dordr). 2015;37(4):9814.

27. Lindsey ML, Goshorn DK, Squires CE, Escobar GP, Hendrick JW, Mingoia JT, et al. Age-dependent changes in myocardial matrix metalloproteinase/tissue inhibitor of metalloproteinase profiles and fibroblast function. Cardiovasc Res. 2005;66(2):410-419.

28. Hanes DS, Weir MR, Sowers JR. Gender considerations in hypertension pathophysiology and treatment. Am J Med. 1996;101(3A):10S-21S.

29. Leening MJ, Ferket BS, Steyerberg EW, Kavousi M, Deckers JW, Nieboer D, et al. Sex differences in lifetime risk and first manifestation of cardiovascular disease: prospective population based cohort study. BMJ. 2014;349:g5992.

30. Song W, Wang W, Dou LY, Wang Y, Xu Y, Chen LF, et al. The implication of cigarette smoking and cessation on macrophage cholesterol efflux in coronary artery disease patients. J Lipid Res. 2015;56(3):682-691.

31. Molawi K, Wolf Y, Kandalla PK, Favret J, Hagemeyer N, Frenzel K, et al. Progressive replacement of embryo-derived cardiac macrophages with age. J Exp Med. 2014;211(11):2151-2158.

32. Abu-Taha M, Rius C, Hermenegildo C, Noguera I, Cerda-Nicolas JM, Issekutz AC, et al. Menopause and ovariectomy cause a low grade of systemic inflammation that may be prevented by chronic treatment with low doses of estrogen or losartan. J Immunol. 2009;183(2):1393-402.

33. Pfeilschifter J, Köditz R, Pfohl M, Schatz H. Changes in proinflammatory cytokine activity after menopause. Endocr Rev. 2002;23(1):90-119. 


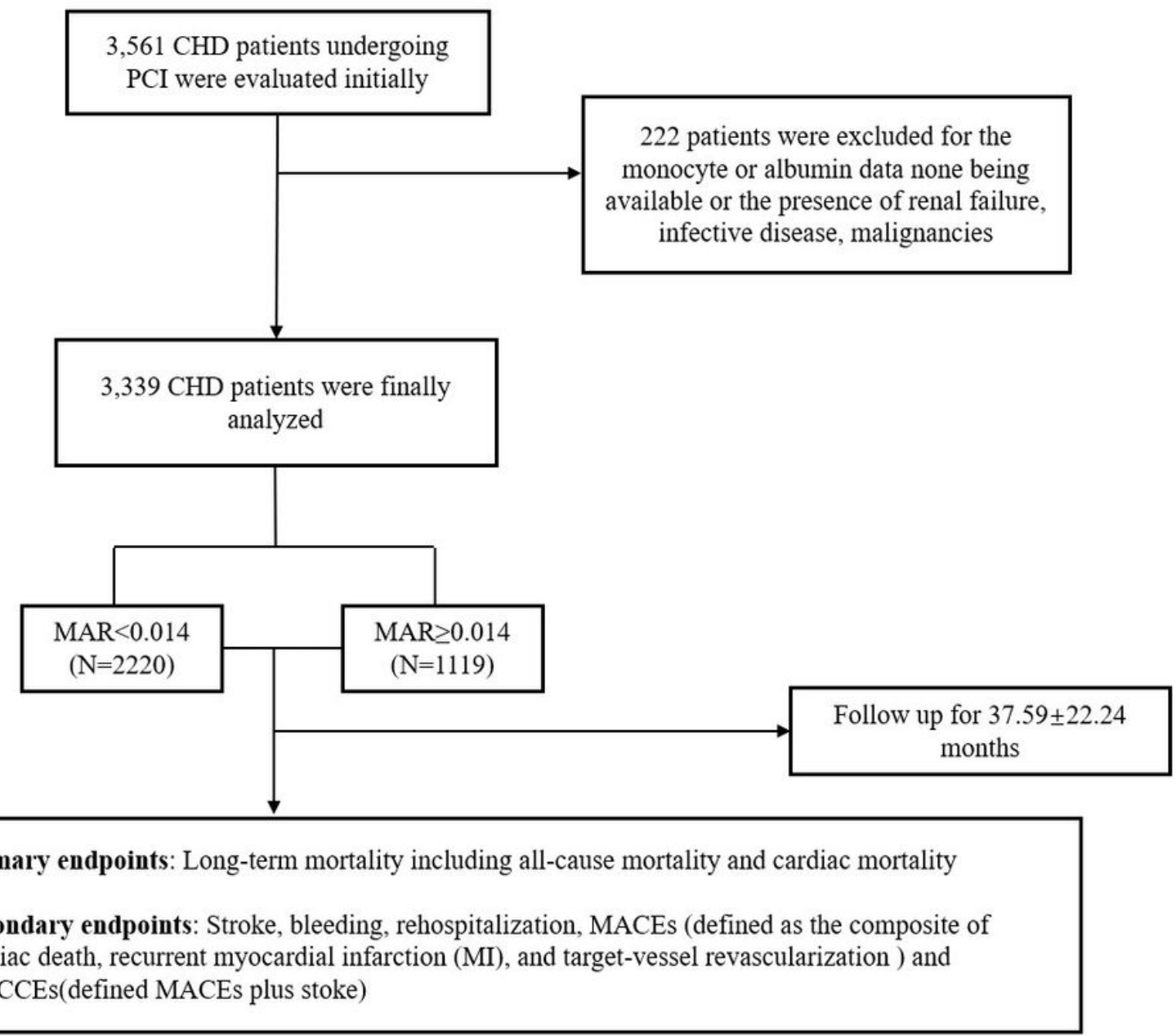

\section{Figure 1}

The flowchart of patients' enrollment 


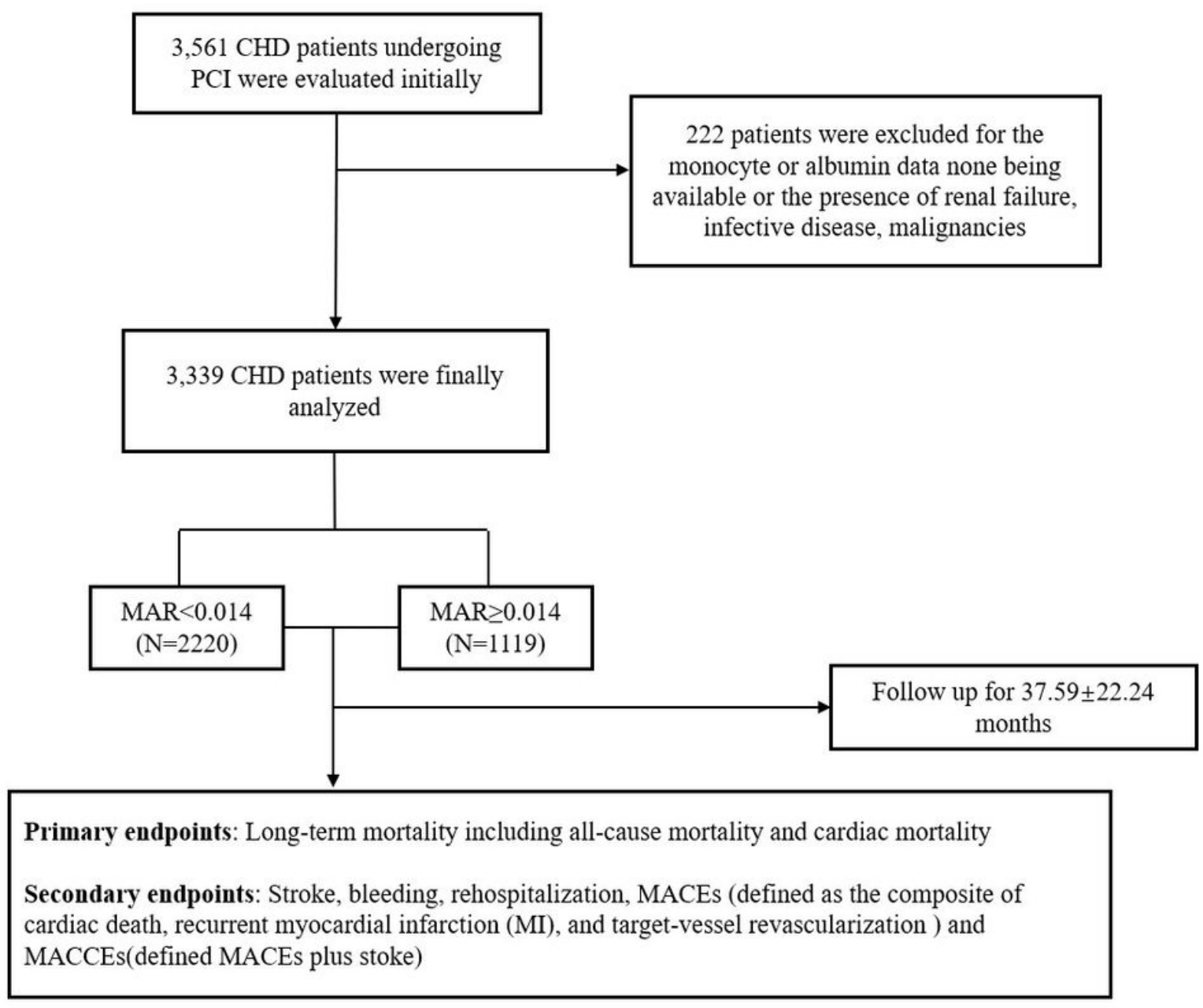

Figure 1

The flowchart of patients' enrollment 


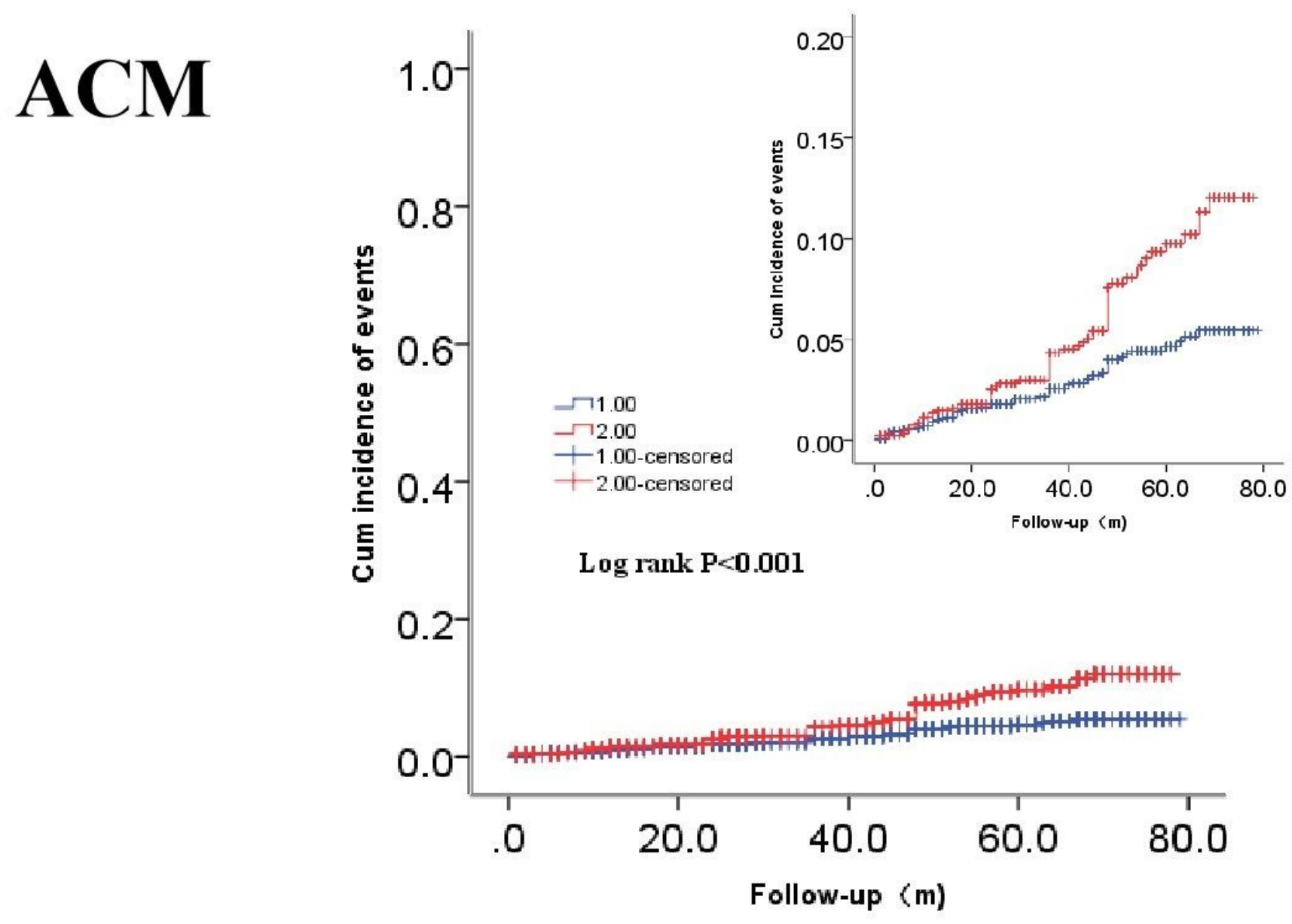

Figure 2

Cumulative Kaplan-Meier estimates of the time to the first adjudicated occurrence of ACM 


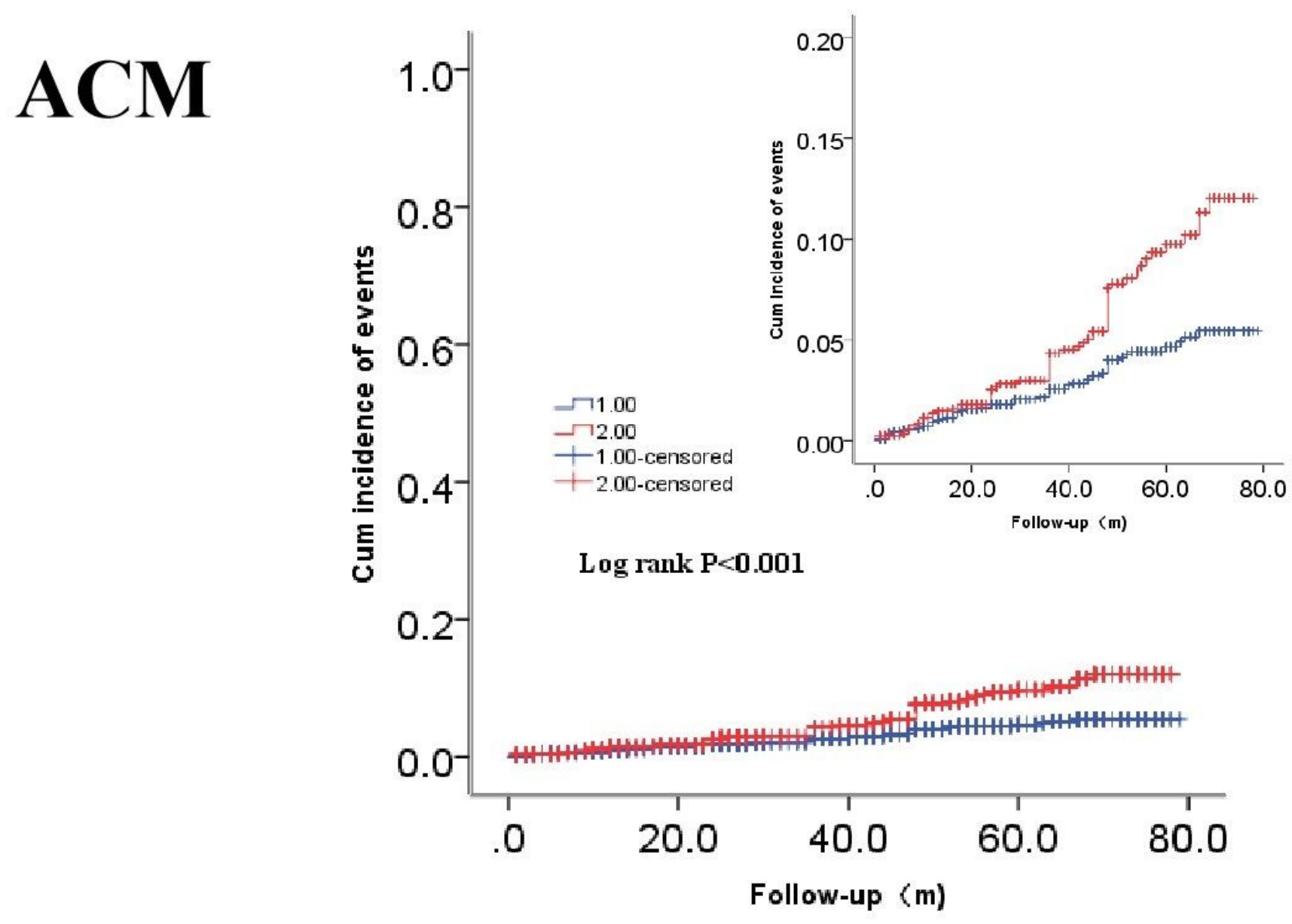

Figure 2

Cumulative Kaplan-Meier estimates of the time to the first adjudicated occurrence of ACM 


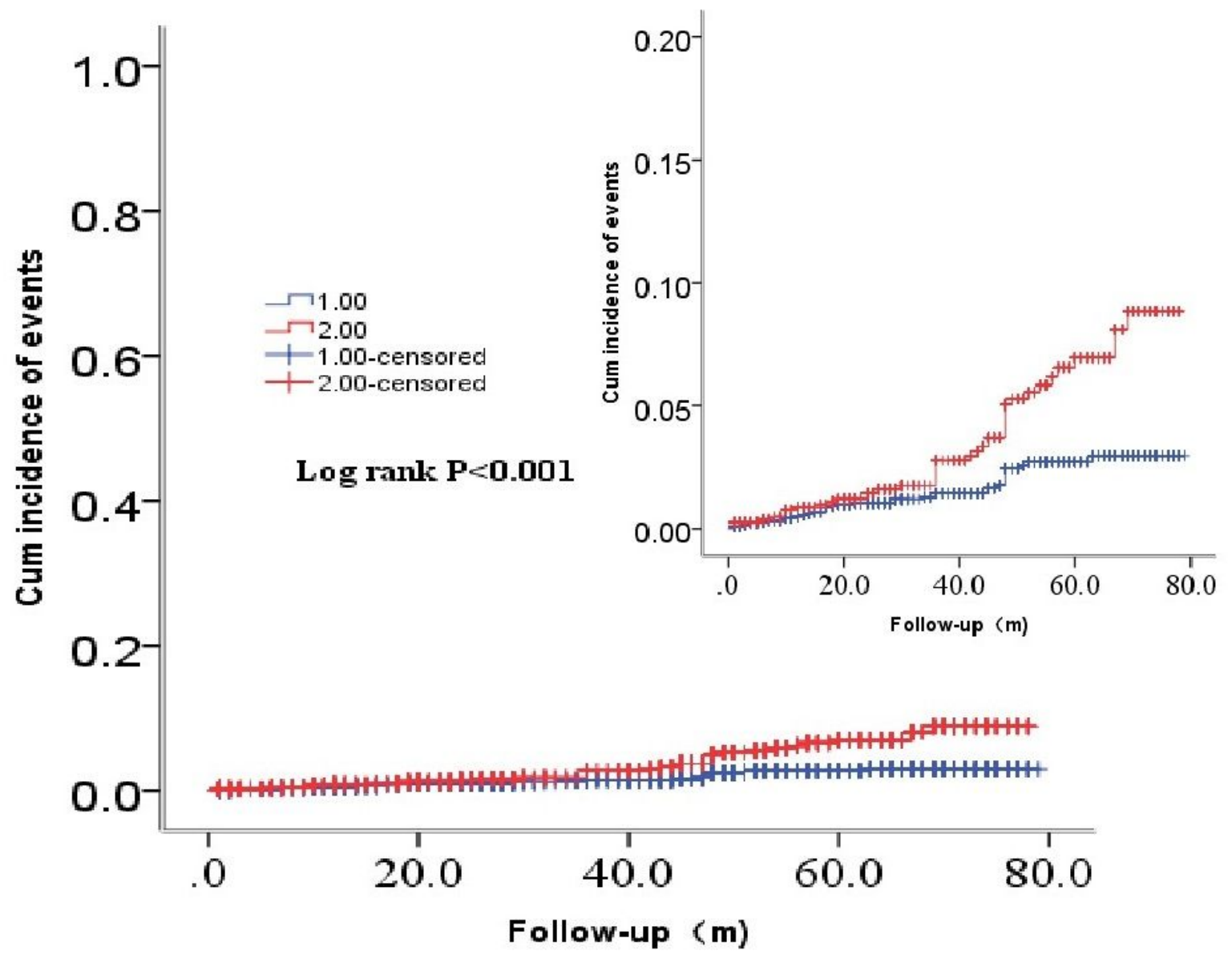

Figure 3

Cumulative Kaplan-Meier estimates of the time to the first adjudicated occurrence of CM In figure 2 and figure 3 , the red line indicates the higher MAR, and blue line indicates the lower MAR. 


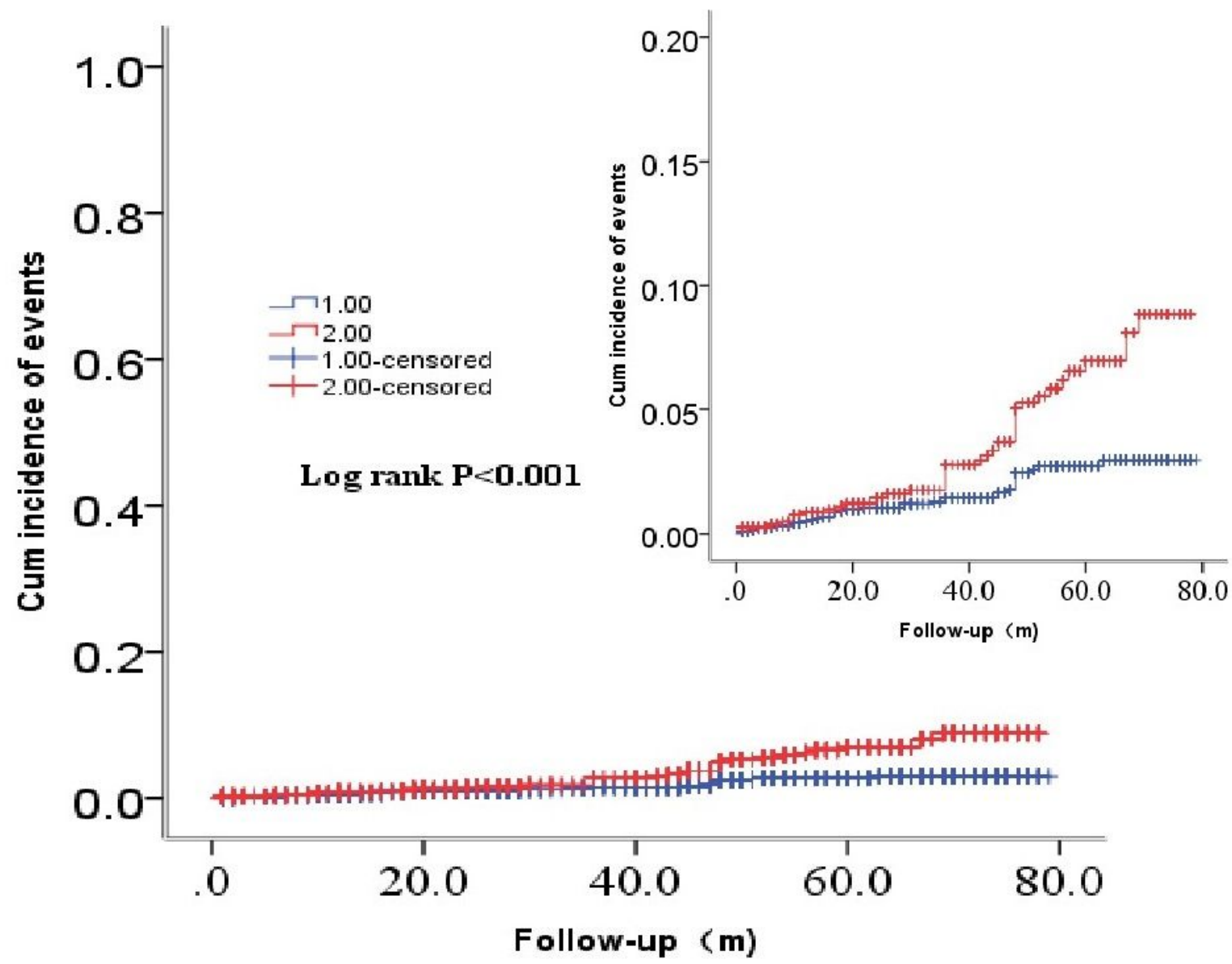

Figure 3

Cumulative Kaplan-Meier estimates of the time to the first adjudicated occurrence of CM In figure 2 and figure 3 , the red line indicates the higher MAR, and blue line indicates the lower MAR. 